\title{
On the Utility of Rate Adaptation for Broadcast/Multicast Media Traffic in 802.11 Networks
}

\author{
Kyungsoo Lee \\ Dept. of Computer Science, \\ University of Massachusetts - Amherst \\ Amherst, MA, USA \\ kslee@cs.umass.edu
}

\author{
Sangki Yun, Yongtae Park, and Hyogon \\ Kim \\ Dept. of Computer Science and Engineering \\ Korea University \\ Seoul, Korea
}

\begin{abstract}
As there is no MAC layer acknowledgment from the receiver for broadcast/multicast transmission in the 802.11 network, using the lowest transmission rate is understandably the safest approach because it minimizes reception error for clients with the worst channel condition. On the contrary, the transmission rate for receivers with good channel conditions is unnecessarily limited. In the case of video streams being broadcast/multicast via 802.11 , this approach will lead to poor received video at all clients.

In this paper, we demonstrate that there is utility in activating rate adaptation for broadcast/multicast traffic. In particular, we implement a system that significantly improves the broadcast video quality in 802.11 network. This is achieved through an approach of joined broadcast/multicast rate adaptation and network-coded retransmission.
\end{abstract}

Categories and Subject Descriptors: C.2.1 [Computer-Communication Networks]: Wireless communication

General Terms: Design, Experiments, Performance, Reliability

Keywords: IEEE 802.11, Broadcast, Multicast, Rate adaptation, Network coding

\section{INTRODUCTION}

As wireless networks are becoming a more important Internet access technology, the demand for support of broadcast and multicast traffic in wireless networks is also increasing. For instance, in video streaming services such as Internet Protocol Television (IPTV), wireless multicasting is useful to make this service scalable. Unfortunately, wireless network protocols such as 802.11 [1] do currently not provide the quality required to multicast transmission of video streams. This is due to the fact that the selected rate for multicast transmissions is fixed (and often set too low for the transmission of video streams [2]) and no acknowledgment and retransmission capabilities are provided. This lack of these capabilities often resulted in unacceptably poor video quality in such cases where the video stream is multicast. In this paper, we propose an extension to the default 802.11 multicast mechanism, an intelligent rate adaptation jointly working with network coded retransmission. In particular, we show with the help of a broadcast

Permission to make digital or hard copies of all or part of this work for personal or classroom use is granted without fee provided that copies are not made or distributed for profit or commercial advantage and that copies bear this notice and the full citation on the first page. To copy otherwise, to republish, to post on servers or to redistribute to lists, requires prior specific permission and/or a fee.

CoNEXT'09 Student Workshop, December 1, 2009, Rome, Italy.

Copyright 2009 ACM 978-1-60558-751-6/09/12 ...\$10.00. video streaming example that rate adaptation in combination with network-coded retransmission can overcome channel-induced picture distortion much better than "safely" relying on the lowest rate.

\section{PROPOSED SYSTEM DESIGN}

The key feature of our proposed BRANC(Broadcast Rate Adaptation with network-coded retransmission) is the joint design of broadcast and multicast rate adaptation and network coded retransmission. These two mechanisms work synergistically, in that rate adaptation creates opportunities for coded transmission that supplements native transmissions, and network-coded retransmission compensates for the increase in loss rate due to the raised transmission rate. We next introduce each component in BRANC.

\subsection{Feedback and Retransmission system}

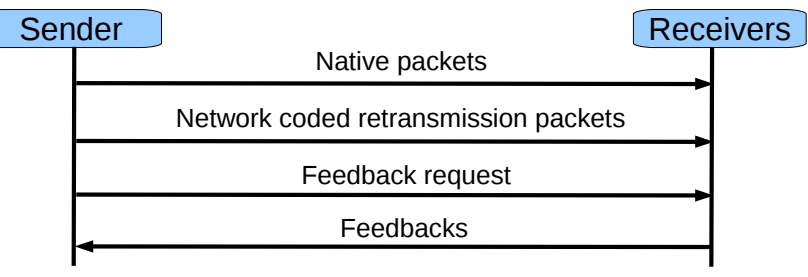

Figure 1: Packet flow of BRANC.

In Fig. 1, we illustrate the process of BRANC.

The sender requests feedback from receivers in $100 \mathrm{~ms}$ intervals. This interval size is chosen to prevent the waste of bandwidth by the frequent transmission of small feedback packets. After receiving the request, receivers send feedback, including their observed loss rate. The sender then decides on the tranmission rate and the number of retransmissions based on this feedback.

The retransmission mechanism for broadcast/multicast traffic is based on [3]. In BRANC, the sender encodes native data packets with random linear combination using network coding. The sender transmits encoded packets after transmitting native data packets. Receivers can derive missed packets from decoding native packets and network-coded packets if sufficient packets for decoding arrive at the receiver. For example, if a sender sent 5 native packets and 3 network-coded packets, a receiver can recover lost packets by decoding received packets when it receives more than 5 out of 8 packets.

\subsection{Rate Adaptation Strategy}

We start with the assumption $r^{i}>\lambda$ for avoiding queueing loss, where $r^{i}$ is the current transmission rate at mode $i$ and $\lambda$ is the 


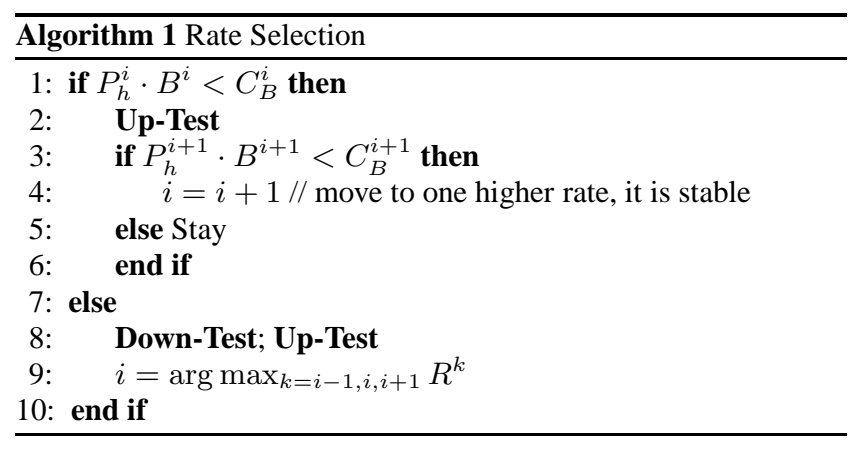

media rate. The rate selection algorithm works as described in Algorithm 1. Periodically, using the receiver reports, the sender determines if the current rate is sustainable and if so, it ventures to one higher rate in the rate hierarchy. In line 1, it checks if the lost amount of data can be filled by network-coded retransmissions using unused bandwidth. $P_{h}^{i}$ is the highest packet loss rate induced by the channel at rate $r^{i}$, as reported by the receivers. The condition holds if the number of lost native frames $P_{h}^{i} \cdot N_{B}^{i}$ at the worst receiver due to channel conditions is smaller than the number of surviving ones among coded frames, i.e., $\left(1-P_{h}^{i}\right) \cdot C_{B}^{i}$, where $N_{B}$ is the number of native packets and $C_{B}$ is the number of coded packets. Namely, the stability condition at rate $r_{i}$ is

$$
\begin{aligned}
& P_{h}^{i} \cdot N_{B}^{i}<\left(1-P_{h}^{i}\right) \cdot C_{B}^{i} \\
\Rightarrow \quad & P_{h}^{i}\left(N_{B}^{i}+C_{B}^{i}\right)<C_{B}^{i}
\end{aligned}
$$

Notice the coding block size is $B^{i}=N_{B}^{i}+C_{B}^{i}$, which leads us to the condition in line 1 . Then we can recover the entire block for the reported $P_{h}^{i}$, and the current rate $r^{i}$ is sustainable. Moreover, it qualifies for the condition to check one higher rate in the next period (line 2, Up-Test). The Up-Test is simply sending one period worth of frames at $r^{i+1}$. Then the rate selection algorithm executed in the next period considers the reports for the increased rate $r^{i+1}$. The same condition is checked in line 3, except for the rate index $i+1$. If this test fails, however, the rate is maintained at $r^{i}$ (line 5) until the next test.

If the current rate is not stable (line 7), we perform two tests, for $r^{i-1}$ and $r^{i+1}$ (line 8). The reason for Down-Test is obvious, but the Up-Test is novel. This is the most important distinction of BRANC in comparison to usual rate adaptation algorithms. We argue that

$$
\begin{array}{ll} 
& P_{h}^{i} \cdot N_{B}^{i}>\left(1-P_{h}^{i}\right) \cdot C_{B}^{i} \\
\Leftrightarrow & P_{h}^{i+1} \cdot N_{B}^{i+1}>\left(1-P_{h}^{i+1}\right) \cdot C_{B}^{i+1}
\end{array}
$$

by ramping up the transmission rate we obtain $C_{B}^{i}<C_{B}^{i+1}$ although it is likely $P_{h}^{i+1}>P_{h}^{i}$ given the same channel condition. So it is possible that when $r^{i}$ is not stable, $r^{i+1}$ is. The Up-Test in line 8 checks this possibility.

In line 9, the result of the two tests is used to check which of the adjacent three rates $\left(r^{i-1}, r^{i}, r^{i+1}\right)$ has the best potential. Let

$$
R^{i} \equiv\left(1-P_{h}^{i}\right) \cdot C_{B}^{i}-P_{h}^{i} \cdot N_{B}^{i}=C_{B}^{i}-P_{h}^{i} \cdot B^{i}
$$

be the residual capacity at $r^{i}$, defined to be the number of excess frames after successful recovery of the given block.

\section{EXPERIMENTAL EVALUATION}

In this section, we evaluate how effectively BRANC protects the broadcast/multicast traffic, through the example of real-time video broadcast. We implemented BRANC on MadWiFi device driver [4] For evaluation, we installed 5 Fujitsu notebooks with Fedora Core 7 [5] in the laboratory of Korea University( 1 sender, 4 receivers.)

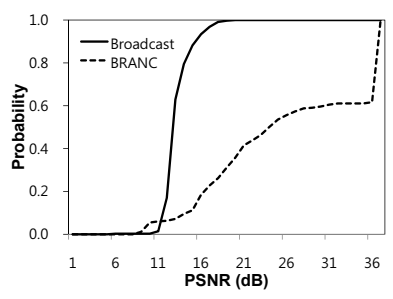

(a) Bad channel.

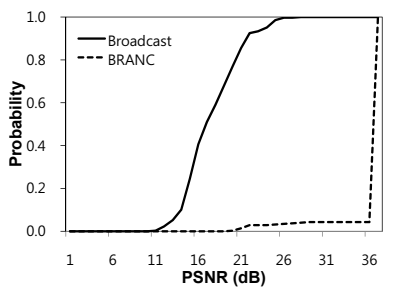

(b) Good channel.
Figure 2: Cumulative PSNR distribution. $(\lambda=12 \mathrm{Mbps})$

We first analyze the worst link of this experiment with a PER of about $15 \%^{1}$ with a transmission rate of $18 \mathrm{Mbps}$, which induces poor quality in the received video. Fig. $2(a)^{2}$ shows the result when the video rate is $\lambda=12 \mathrm{Mbps}$. In this experiment, 802.11 standard broadcast uses $18 \mathrm{Mbps}$ transmission rate to avoid queueing loss. We observe that most video frames have less than 15dB PSNR in the 802.11 broadcast. BRANC performs much better than the 802.11 broadcast. Fig. 2(a) shows that it only sporadically falls below 20dB PSNR, and more than $40 \%$ of frames have the maximum PSNR. It demonstrates the potential of rate adaptation that if supplemented by network-coded retransmission it can actually protect media traffic better than conservatively picking the lowest rate that satisfies the media rate.

In addition, we analyze the best performing link which showed a PER of about $4 \%$ at a transmission rate of $18 \mathrm{Mbps}$. This link is that even the $54 \mathrm{Mbps}$ rate experiences less than $10 \%$ loss in this link. The result with the link is shown on Fig. 2(b) when $\lambda=12 \mathrm{Mbps}$. In this case, BRANC improves the video quality as well.

\section{CONCLUSION}

In this paper, we proposed a joint design approach that combines rate adaptation and network coded retransmission. Through the broadcast video example, we demonstrated that the novel approach offers a significantly better alternative, with a video quality far exceeding that of the native 802.11 broadcast.

In the future, we plan to refine the joint algorithm so that it uses smaller bandwidth by adaptively controlling the number of coded supplementary transmissions, creating a larger room for more media calls or competing traffic.

\section{ACKNOWLEDGEMENT}

This research is supported by the Ubiquitous Computing and Network(UCN) Project, Knowledge and Economy Frontier R\&D Program of the Ministry of Knowledge Economy(MKE) in Korea as a result of UCN's subproject 09C1-C1-20S.

\section{REFERENCES}

[1] IEEE, Part 11: Wireless LAN Medium Access Control (MAC) and Physical Layer (PHY) Specifications. IEEE Std 802.11-1999, Aug. 1999.

[2] S. Choi, N. Choi, Y. Seok, T. Kwon, and Y. Choi,"Leader-based Rate Adaptive Multicasting for Wireless LANs," IEEE Global Telecommunications Confernce (Globecom) 2006.

[3] D. Nguyen, T. Nguyen and B. Bose, "Wireless Broadcasting Using Network Coding," in proceedings of NetCod 2007.

[4] MADWiFi: Multiband Atheros Driver for WiFi. http://madwifi.org

[5] Fedora Project. http://www.fedoraproject.org

\footnotetext{
${ }^{1}$ The PER is observed by measuring individual links.

${ }^{2}$ The $y$-axis in the graph shows an empirical CDF.
} 